\title{
Elastin (ELM) gene point mutation in patients with inguinal hernia
}

\author{
Consuelo Junqueira Rodrigues, Jin Hwan Yoo and Aldo Junqueira Rodrigues Junior \\ Universidade de São Paulo, Hospital das Clínicas, Faculdade de Medicina, Departamento de Cirurgia, \\ São Paulo, SP, Brazil.
}

\begin{abstract}
Groin hernias emerge at the myopectineal orifice of Fruchaud which is closed off by the fascia transversalis. Our previous studies showed structural and quantitative changes of the fascia transversalis elastic fibers of inguinal hernia patients and elderly people. The present study used single-strand conformation polymorphism (SSCP) elastin (analysis to investigate the 34 exons of the ELN gene of 49 inguinal hernia patients ( 7 females, 42 males aged $58.7 \pm 19.82$ years) and 75 non-herniated controls ( 35 females, 40 males aged $46.2 \pm 14.32$ years). We found that 47 patients and 24 controls had an abnormal exon 20 pattern caused by a g28197A > G missense mutation leading to an S422G amino acid substitution in the elastin hydrophobic domain. The g28197A > G allele frequency was $0.71 \pm 0.045$ in hernia patients and $0.21 \pm 0.030$ in controls and 23 patients and 7 controls were g28197A $>$ G homozygous and 24 patients and 17 controls were heterozygous. This point-mutation showed a statistically significant association with inguinal hernia, chi-squared being $46.89(p<0.001)$ and the odds ratio 49.93 (95\% confidence interval of $\cong 11$ to 223). These results indicate that the g28197A > G mutation is involved in the genesis of inguinal hernia (possibly due to abnormal elastic fiber production) and explains impaired fascia transversalis function.
\end{abstract}

Key words: fascia transversalis, extracellular matrix, elastic fibers, elastin gene, inguinal hernia.

Received: October 13, 2004; Accepted: July 26, 2005.

All groin hernias emerge at the myopectineal orifice of Fruchaud which is closed off by the fascia transversalis, a connective tissue composed of a framework of elastic and collagen fibers, which supports the abdominal tension forces.

The elastic fibers are composed of microfibrils and elastin and are responsible for the resilience of the fascia transversalis. Research by our group has shown age-related structural changes (breaching and wrapping) in the elastic fibers, with ultrastructural studies showing a large decrease in the quantity of microfibril component ( Rodrigues Jr. et al., 1990; Quintas et al., 2000). Some studies have shown reduced amounts of collagen in the fascia transversalis of patients with direct inguinal hernia compared to those with indirect hernia, suggesting that the development of some hernias might be influenced by disturbances in collagen metabolism (Pans et al., 2001; Rodrigues Jr et al., 2002). Taken together, these results indicate that there is a loss of tensile resistance and elasticity in the fascia transversalis tissue which may explain the high incidence of inguinal hernia after the fifth decade of life.

Send correspondence to Consuelo J Rodrigues. Universidade de São Paulo, Hospital das Clínicas, Faculdade de Medicine, Departamento de Cirurgia, Av. Dr. Arnaldo 455, sala 1304, 01246-903 São Paulo, SP, Brazil. E-mail: consuelo@lim02.fm.usp.br.
Mutations in the elastin (ELN) gene may be responsible for disorders of the elastic fiber system because they have been observed in cases of supravalvular aortic stenosis and autosomal dominant cutis laxa, individuals with either of these conditions may show smooth and soft skin, hernias, gastrointestinal diverticula and genital prolapse (Ewart et al., 1994; Li et al., 1997; Meng et al., 1998; Tassabehji et al., 1998). These reports and our previous studies plus the findings by Pans et al. (2001) that there were biomechanical and structural changes in the fascia transversalis from both the herniated and the non-herniated sides of individual hernia sufferers suggests that hernias may represent a localized somatic expression of a generalized alteration in collagen and elastic fibers, possibly linked to as yet unknown genetic factors. To explore the possibility of mutations in the ELN gene contributing to hernia we screened individuals with inguinal hernia and matched controls without inguinal hernia.

Genomic DNA was obtained from peripheral blood leukocytes of 49 patients ( 7 females, 42 males aged $58.7 \pm 19.82$ years) randomly selected from those presenting with inguinal hernia and from 75 non-herniated controls (35 females, 40 males aged $46.2 \pm 14.32$ years). This study was performed according to protocols approved by the ethics board of our institution and all the individuals involved provided informed consent. We initially screened 
for $E L N$ gene mutations using single-strand conformation polymorphism (SSCP) analysis (Orita et al., 1989). Each of the 34 exons of the $E L N$ gene was amplified using intronic primers as previously described by Tassabehji et al. (1997) and the PCR products separated by overnight electrophoreses on non-denaturing $10 \%(\mathrm{w} / \mathrm{v})$ polyacrylamide gels (49:1 acrylamide:N, N' bisacrylamide) at $4{ }^{\circ} \mathrm{C}$ and a constant $550 \mathrm{~V}$, the products being detected by silver staining. Whenever an abnormal banding pattern was observed the relevant exon was directly sequenced using an ABI Prism 377 sequencer (Perkin Elmer). Sequences were aligned by using the basic local alignment search tool (BLAST) (Altschul et al., 1999) using the nucleotide sequence data deposited in GenBank under access number NT007758.10.

Our initial SSCP analysis examined the $34 E L N \mathrm{f}$ gene exons of 19 inguinal hernia patients and 16 nonherniated controls and detected abnormal banding mobility in exon 20 of all the 19 patients and 11 of the controls. Sequence analysis revealed an exon 20 missense mutation consisting of a single g28197A $>$ G nucleotide substitution corresponding to a S422G substitution at the protein level (NP_000492). These results prompted us to sequence the $E L N$ exon 20 from an additional group of 30 inguinal hernia patients and 59 non-herniated controls. In all, the g28197A > G missense mutation was present in 47 out of 49 herniated patients and 24 out of 75 non-herniated controls and this led to an $\mathrm{S} 422 \mathrm{G}$ amino acid substitution in the elastin hydrophobic domain. This point-mutation showed a statistically significant association with inguinal hernia, chi-squared being $46.89(\mathrm{p}<0.001)$ and the odds ratio $49.93(95 \%$ confidence interval $=11$ to 223$)$. We found that 23 hernia patients and 7 controls were homozygous for the mutant allele and 24 patients and 17 controls were heterozygous. The mutant allele frequency was $0.71 \pm 0.045$ in hernia patients and $0.21 \pm 0.030$ in the controls.

The $E L N$ f gene exon $20 \mathrm{~g} 28197 \mathrm{~A}>\mathrm{G}$ missense point mutation is present at a significantly higher frequency in inguinal hernia patients than in non-herniated controls. Normal tropoelastin is rich in non-polar amino acids which produce the hydrophobic domain required for the elastic property of the fiber but the g28197A > G missense mutation changes non-polar amino acids to uncharged ones resulting in hydrophobic changes in the tropoelastin. Variability in the amino acid sequence may change the tropoelastin conformation and produced defective elastin fibers, abnormal fibrillogenesis or an altered response to enzymatic degradation (Parks and Deak, 1990). It appears that the accumulation of damaged elastic fibers which occurs in the fascia transversalis of elderly people and patients with inguinal hernia may be a consequence of mutations in $E L N$ gene. Tassabehji et al. (1998) detected a frameshift mutation in exon 32 of the $E L N$ gene of a patient affected by dominant autosomal cutis laxa, this mutation produced a defective tropoelastin protein which altered the architecture of elastic fibers and produced skin with a prematurely aged appearance.

Our results favor the hypothesis that the $E L N$ f gene exon 20 g28197A > G missense mutation leads to the production of abnormal elastic fibers and the loss of fascia transversalis function and thus plays a role in the genesis of inguinal hernia.

\section{References}

Altschul SF, Gish W, Miller W, Myers EW and Lipman DJ (1999) Basic local alignment search tool. Bioinformatics 15:862863.

Ewart AK, Jin W, Atkinson D, Hurst J, Reardon W, Bruch M and Read AP (1994) Supravalvular aortic stenosis associated with a deletion disrupting the elastin gene. J Clin Invest 93:1071-1077.

Li DY, Toland AE, Boak BB, Atkinson DL, Ensing GJ, Morris CA and Keating MT (1997) Elastin point mutations cause an obstructive vascular disease, supravalvular aortic stenosis. Hum Mol Genet 6:1021-1028.

Meng X, Lu X, Li Z, Green E, Massa H, Trask BJ, Morris CA and Keating MT (1998) Complete physical map of the common deletion region in Williams syndrome and identification and characterization of three novel genes. Hum Genet 103:590599.

Orita M, Iwahana H, Kanazawa K, Hayashi K and Sekiya T (1989) Detection of polymorphisms of human DNA by gel electrophoresis as single-strand conformation polymorphisms. Proc Natl Acad Sci USA 86:2766-2770.

Pans A, Albert A, Lapiere CM and Nusgens B (2001) Biochemical study of collagen in adult groin hernias. J Surg Res 95:107-113.

Parks WC and Deak SB (1990) Tropoelastin heterogeneity: Implications for protein function and disease. Am J Respir Cell Mol Biol 2:399-406.

Quintas M, Rodrigues CJ, Yoo JH and Rodrigues Jr AJ (2000) Age related changes in the elastic fiber system of the interfoveolar ligament. Rev Hosp Clin Fac Med S Paulo 55:83-86.

Rodrigues Jr AJ, Rodrigues CJ, Cunha ACP and Yoo JH (2002) Quantitative analysis of collagen and elastic fibers in transversalis fascia in direct and indirect inguinal hernia. Rev Hosp Clin Fac Med S Paulo 57:265-270.

Rodrigues Junior AJ, Castro de Tolosa EM and Ferraz de Carvalho CA (1990) Electron microscopic study on the elastic and elastic related fibers in the human fascia transversalis at different ages. Gegenbaurs morphol Jahrb 136:645-652.

Tassabehji M, Metcalfe K, Donnal D, Hurst J, Reardon W, Bruch $M$ and Read AP (1997) Elastin: Genomic structure and point mutations in patients with supravalvular aortic stenosis. Hum Mol Gen 6:1029-1036.

Tassabehji M, Metcalfe K, Hurst J, Ashcroft GS, Kielty C, Wilmot C, Donnai D, Read AP and Jones CJ (1998) An elastin gene mutation producing abnormal tropoelastin and abnormal elastic fibers in a patient with autosomal dominant cutis laxa. Hum Mol Genet 7:1021-1028.

Associate Editor: Angela M. Vianna-Morgante 